\title{
Towards higher resolution in Magnetic Force Microscopy
}

\author{
Leon Abelmann ${ }^{1}$, Arnout van den Bos ${ }^{1}$, and Cock Lodder ${ }^{1}$ \\ Systems and Materials for Information Storage \\ University of Twente, 7500 AE Enschede, The Netherlands
}

\begin{abstract}
In this chapter Magnetic Force Microscopy is treated in detail. The emphasis is on high resolution and the design and realisation of MFM tips. ${ }^{1}$
\end{abstract}

\section{Principle of MFM}

The technique of Magnetic Force Microscopy has been discussed extensively in literature $[8,20,12]$, so we will restrict ourselves to a short description. The principle of Magnetic Force Microscopy is very much alike that of Atomic Force Microscopy - some even dare to mention that MFM is just an AFM with a magnetic tip, much to the dislike of MFM developers because in an MFM much smaller forces are measured. In essence it is true however, and every MFM is capable of AFM as well (the other way round is not true in general).

In an MFM the magnetic stray field above a very flat specimen, or sample, is detected by placing a small magnetic element, the tip, mounted on cantilever spring very close to the surface of the sample (figure 1). Typical dimensions are a cantilever length of $200 \mu \mathrm{m}$, tip length of $4 \mu \mathrm{m}$ and diameter of $50 \mathrm{~nm}$ and a distance from the surface of $30 \mathrm{~nm}$. The force on the magnetic tip is detected by measuring the displacement of the end of the cantilever, usually by optical means. The forces measured in typical MFM applications are in the order of $30 \mathrm{pN}$, with typical cantilever deflections in the order of nanometers.

An image of the magnetic stray field is obtained by slowly scanning the cantilever over the sample surface, in a raster-like fashion. Typical scan area's are from 1 up to $200 \mu \mathrm{m}$ with imaging times in the order of 5-30 minutes.

\subsection{Mode of operation}

The force $F$ excerted on the tip by the stray field of the sample has two effects on the cantilever deflection. In the first place the cantilever end is deflected towards or away from the sample surface by a distance $\Delta z$ :

\footnotetext{
1 This chapter is accompanied by a webpage http://www.el.utwente.nl/smi/mfmchapter/, where you can find errata and links for further reading
} 


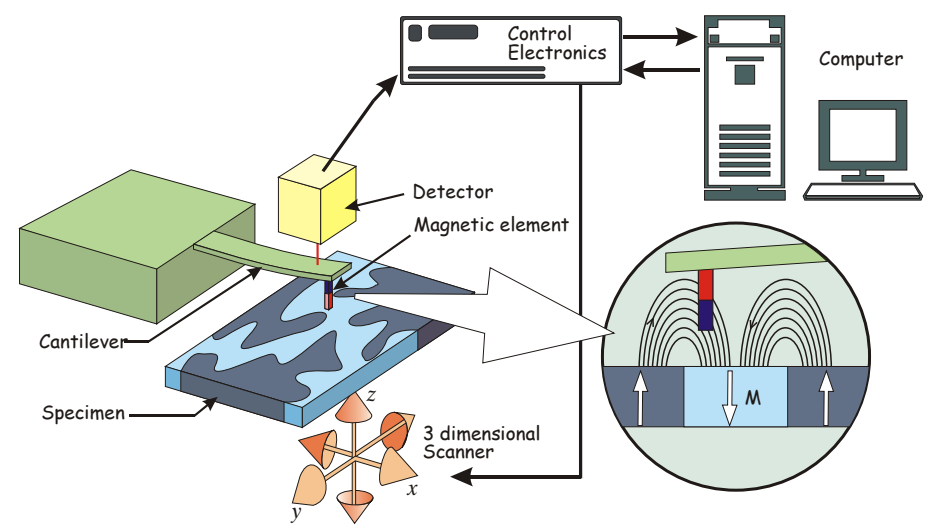

Fig. 1. Principle of Magnetic Force Microscopy

$$
\Delta z=F_{z} / c[m]
$$

Where $c$ is the cantilever spring constant in $z$-direction $[N / m]$. This deflection can be measured using soft, usually $\mathrm{Si}_{3} \mathrm{~N}_{4}$, cantilevers with spring constants in the order of $0.01-0.1 \mathrm{~N} / \mathrm{m}$. When measuring the deflection, we speak about static mode MFM .

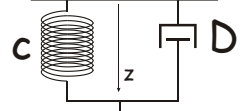

m

Fig. 2. The cantilever can be modelled as a damped harmonic oscillator

For small deflections, the cantilever can be considered as a damped harmonic oscillator, which can be modelled by an ideal spring $c[N / m]$, mass $m[\mathrm{~kg}]$ and damper $D[N s / m]$ (Figure 2) [3]. When applying an oscillating force $F_{z}=F_{0} \cos (\omega t)$ on the end of the cantilever, the resulting displacement is harmonic as well, but has a phase shift for $\omega>0, z=z_{0} \cos (\omega t+\theta(\omega))$. It is convenient to desribe the relation between force and displacement in the Laplace domain ${ }^{2}$

$$
\frac{\widehat{Z}}{\widehat{F}}=\frac{1}{c+s D+m s^{2}}[m / N]
$$

${ }^{2 \widehat{F}}(s)=\int_{-\infty}^{+\infty} f(t) e^{-s t} d t$ 
Using the natural resonance frequency $\omega_{n}$ and unitless damping factor $\delta$

$$
\begin{aligned}
\omega_{n} & =\sqrt{c / m}[2 \pi / s] \\
\delta=\frac{D}{2 \sqrt{m c}} & =\frac{D \omega_{n}}{2 c}
\end{aligned}
$$

we can rewrite (2) into

$$
\frac{\widehat{Z}}{\widehat{F}}=\frac{1}{m \omega_{n}^{2}+2 \delta \omega_{n} m s+m s^{2}}[m / N]
$$

For underdamped systems $(\delta<1)$, this system has poles at

$$
s_{1}, s_{2}=-\delta \omega_{n} \pm i \omega_{n} \sqrt{1-\delta^{2}}=-\delta \omega_{n} \pm i \omega_{d}
$$

The term $\omega_{d}=\omega_{n} \sqrt{1-\delta^{2}}$ is referred to as the damped natural frequency. For MFM in dynamic mode cantilevers with very small damping $(\delta \ll 0.01)$ are used and $\omega_{d} \approx \omega_{n}$. In MFM it is custom to talk about the quality factor of resonance $Q$, instead of the damping factor. $Q$ is proportional to the ratio between the energy stored in the cantilever and the energy lost per cycle:

$$
Q=2 \pi \frac{\text { Energy stored in cantilever }}{\text { Energy lost per cycle }}=2 \pi \frac{\frac{1}{2} c z_{0}^{2}}{\pi D z_{0}^{2} \omega_{n}}=\frac{c}{D \omega_{n}}=\frac{1}{2 \delta}
$$

Using $Q(5)$ becomes:

$$
\frac{\widehat{Z}}{\widehat{F}}=\frac{1}{m \omega_{n}^{2}+\frac{\omega_{n} m}{Q} s+m s^{2}}[m / N]
$$

From (8) we can calculate the amplitude $z_{0}$ of the cantilever vibration when driven at a frequency $\omega[2]$

$$
z_{0}=\frac{F_{0} / m}{\sqrt{\left(\omega_{n}^{2}-\omega^{2}\right)^{2}+\left(\omega \omega_{n} / Q\right)^{2}}}
$$

and the phase shift $\theta$ between the force and the deflection (Also see figure 3

$$
\theta=\tan ^{-1}\left(\frac{\omega \omega_{0}}{Q\left(\omega_{0}^{2}-\omega^{2}\right)}\right)
$$

In MFM, the force on the magnetic tip increases when it approaches the sample, so it is as if there is a second spring with a spring constant of $\partial F / \partial z$ 
attached to the cantilever. In the case that the cantilever deflection so small that $\partial F / \partial z$ can be considered a constant, this results in a change in natural resonance frequency $f_{n}=\omega_{n} / 2 \pi$ of the cantilever

$$
\begin{array}{r}
f_{n}^{\prime}=f_{n} \sqrt{1-\frac{\partial F_{z} / \partial z}{c}}[H z] \\
\Delta f=f_{n}^{\prime}-f_{n} \approx-\frac{f_{n}}{2 c} \frac{\partial F_{z}}{\partial z}[H z]
\end{array}
$$

The approximation is accurate for $\Delta f \ll f_{n}$, which is always the case in MFM. Note the sign of $\Delta f$. In the above it is assumed that the positive $z$ direction is pointing away from the surface. When the tip is attracted towards the sample, the force therefore is negative, and the force derivative is positive. So for attracting forces the resonance frequency of the cantilever decreases. Please note that expression (11) is only valid for small vibration amplitudes. When $\partial F / \partial z$ cannot be considered constant, the vibration contains higher harmonics and more eloborate, and even numerical methods are needed to calculate the resonance frequency shifts.

When we measure the resonance frequency of the cantilever, we speak about dynamic mode MFM (indicated by frequency in fig 3). In this mode the cantilever is usually forced to resonate at an amplitude of 10-30 nm, so that an accurate detection of the very small frequency shifts is possible (typically $3 \mathrm{~Hz}$ on $80 \mathrm{kHz}$ ). In this mode a control circuit is needed which matches the beat frequency of the actuator that drives the cantilever (e.g. a piezo), with the actual resonance frequency. Very often a phase locked loop (PLL) circuit is used, which keeps the phase difference between the driving signal and the measured deflection of the cantilever at approximately $90^{\circ}$. This control circuit adds additional noise to the measurement signal. For small signals it is therefore sometimes preferable to fix the frequency of the driving signal to $f_{n}$ and measure the phase difference between the driving signal and the measured cantilever deflection (indicated by phase in fig 3). The phase shift, which is in the order of a few degrees, strongly depends on the damping of the cantilever, which on its turn again is a function of many parameters. The phase signal is therefore not really suitable for quantitative analysis.

An even simpler dynamic detection mode is to drive the cantilever offresonance. A change in resonance frequency will result in change in vibration amplitude (amplitude in fig 3). Even though this amplitude mode works fine for AFM, it gives very poor results for MFM because the amplitude variations are small compared to the noise. Moreover the response of the cantilever to a change in force is slow when the quality factor of resonance is high, which is for instance the case in vacuum[2]. Therefore this mode is not used very often.

Fundamentally there is no difference in sensitivity between the static mode and dynamic mode, because both modes use the same measurement geometry. 


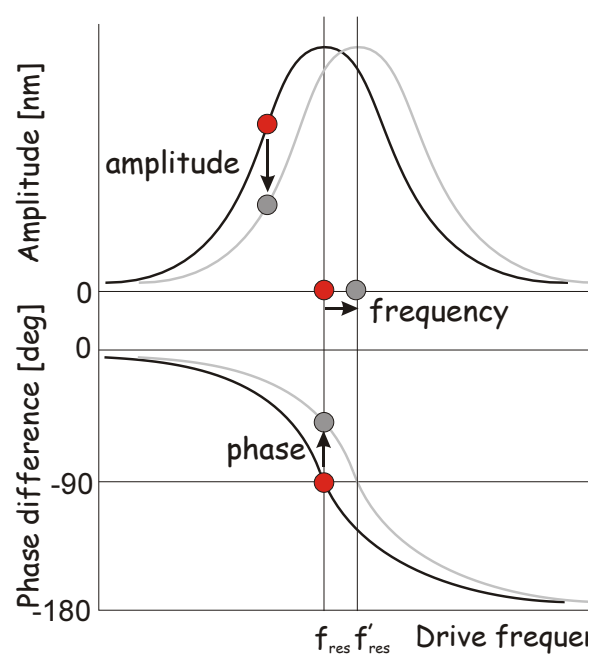

Fig. 3. A change of the magnetic force on the tip results in a change in resonance frequency of the cantilever, which can be detected in different ways.

For a number of reasons, to which we will come back later, the dynamic mode often gives better results however.

\subsection{Image formation}

To calculate the force on the magnetic tip, we have start with the calculation of the energy $U$ of the tip/sample system. The gradient of this energy then gives us the force vector. For MFM we are particularly interested in $\partial U / \partial z$.

We have two ways to calculate $U$. One can either calculate the energy of the magnetic tip in presence of the sample stray field or the energy of the magnetic sample in presence of the tip stray field[12]. In both cases we have to integrate the inproduct of magnetic field and magnetisation over the area where the magnetisation is not zero:

$$
U=-\mu_{0} \int_{\text {tip }} \boldsymbol{M}_{\text {tip }} \boldsymbol{H}_{\text {sample }} d V=-\mu_{0} \int_{\text {sample }} \boldsymbol{M}_{\text {sample }} \boldsymbol{H}_{\text {tip }} d V
$$

Which method is more convenient, depends on the problem which is to be analysed. One usually takes that form for which the stray field calculation is more easy to perform.

When discussing image formation and resolution, it is convenient to do that in the spatial frequency domain- a method commonly used in magnetic recording theory. We therefore decompose the sample magnetisation $\boldsymbol{M}$ in the 
sample plane $(x, y)$ into their Fourier components, leaving the $z$ component untransformed:

$$
\widehat{\boldsymbol{M}}\left(k_{x}, k_{y}, z\right)=\int_{-\infty}^{\infty} \int_{-\infty}^{\infty} \boldsymbol{M}(x, y, z) e^{-i\left(x k_{x}+y k_{y}\right)} d x d y
$$

The relation between the wavelength of a certain component $\lambda$ and the Fourier components is

$$
\begin{gathered}
\boldsymbol{k}=\left(k_{x}, k_{y}\right) \\
k_{x(y)}=\frac{2 \pi}{\lambda_{x(y)}}
\end{gathered}
$$

The stray field of the sample generated by this magnetisation distribution can be calculated by means of a Laplace transform. For a thin film with thickness $t$ one can obtain with some patience [33]

$$
\left(\begin{array}{c}
\widehat{H}_{x}\left(k_{x}, k_{y}, z\right) \\
\widehat{H}_{y}\left(k_{x}, k_{y}, z\right) \\
\widehat{H}_{z}\left(k_{x}, k_{y}, z\right)
\end{array}\right)=\left(\begin{array}{c}
-i k_{x} /|\boldsymbol{k}| \\
-i k_{y} /|\boldsymbol{k}| \\
1
\end{array}\right) \frac{1}{2}\left(1-e^{|\boldsymbol{k}| t}\right) e^{-|\boldsymbol{k}| z} \widehat{\sigma}_{e f f}(\boldsymbol{k})
$$

Where $\widehat{\sigma}_{\text {eff }}(\boldsymbol{k})$ is an effective surface charge distribution. It expresses the property of the Laplace transformation that the stray field at height $z$ above the sample surface is fully determined by the stray field at height $z=0$. The effective surface charge distribution can be seen as a sheet of charges at the sample surfaces, which causes the same stray field as the more complex charge distribution within the sample itself ${ }^{3}$. $\equiv$

For a sample with perpendicular magn 7 ation $\left(M_{x}=0, M_{y}=0\right)$, $\sigma_{e f f}(x, y)$ simply equals the surface charge density $\sigma$.

$$
\widehat{\sigma}_{e f f}(\boldsymbol{k})=\widehat{M}_{z}(\boldsymbol{k})=\widehat{\sigma}(\boldsymbol{k})
$$

For a sample with an in-plane magnetisation (so $M_{z}=0$ ) we only have volume charges $\rho(x, y)$. If the magnetisation is constant over the film thickness $\left(\partial M_{x} / \partial z=0, \partial M_{y} / \partial z=0\right)$ the effective surface charge distribution becomes

$$
\widehat{\sigma}_{e f f}(\boldsymbol{k})=-\frac{i \boldsymbol{k}}{|\boldsymbol{k}|} \cdot \widehat{\boldsymbol{M}}(\boldsymbol{k})=\frac{\widehat{\rho}(\boldsymbol{k})}{|\boldsymbol{k}|}
$$

For more complex situations, every magnetic charge in the sample has to be transformed, which results in rather lengthly expressions, and this method 


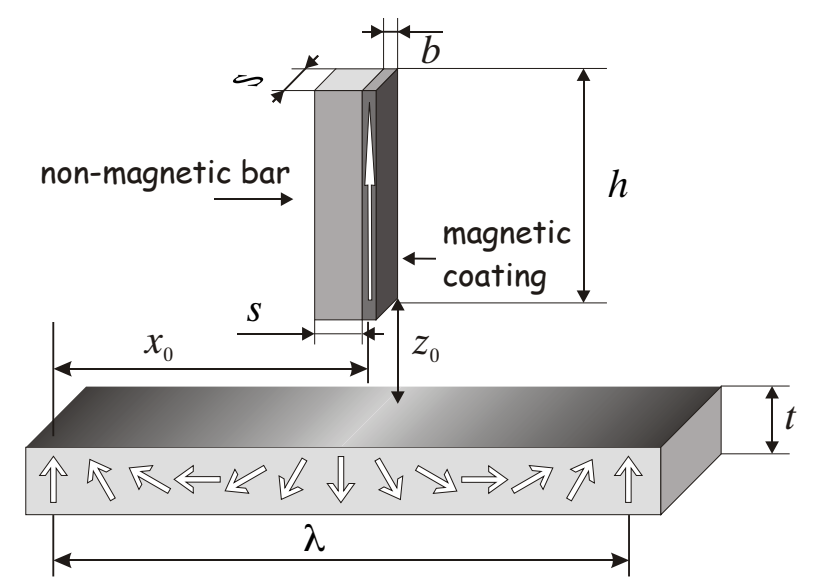

Fig. 4. The ideal MFM tip has a bar shape and a magnetisation fixed along its axis

looses its power. In that case it might be easier to calculate the stray field from the tip.

Assuming a known effective surface charge distribution, we can now calculate the energy of the tip/sample system by combining (13) and (17). The only thing left unknown is the magnetisation distribution in the MFM tip, which can be very complex. We will restrict ourselves however to the bar type tip with a magnetisation fixed along the $z$-axis (Figure 4), in the first place because this is the ideal MFM tip shape [21] and in the second place because it results in very illustrative closed-form equations. The procedure to obtain the force $F_{z}$ involves a simple integral of the stray field over the recangular tip volume, and taking $\partial U / \partial z[21]$ :

$$
\begin{array}{r}
\widehat{F}_{z}(\boldsymbol{k}, z)=-\mu_{0} M_{t} \cdot b \operatorname{sinc}\left(\frac{k_{x} b}{2}\right) \cdot S \operatorname{sinc}\left(\frac{k_{y} S}{2}\right) \\
\times\left(1-e^{-|\boldsymbol{k}| h}\right)\left(1-e^{-|\boldsymbol{k}| t}\right) e^{-|\boldsymbol{k}| z} \widehat{\sigma}_{e f f}(\boldsymbol{k})
\end{array}
$$

Where $M_{t}$ is the tip magnetisation $[A / m], b \times S$ the tip cross section, $h$ the tip height, $t$ the film thickness and $z$ the tip/sample distance (all in $[m])^{4}$. This relation between the force and the effective surface magnetisation is often called the tip transfer function (TTF).

Allthough (20) is complex, it is not difficult to understand. We see that the force is proportional to the tip magnetisation and the magnetic charge

\footnotetext{
${ }^{3}$ The $\widehat{\sigma}_{\text {eff }}$ introduced here differs by a factor of 2 from the effective surface charge distribution defined in $[33,21]$

${ }^{4} \operatorname{sinc}(x)=\sin (x) / x$
} 
density $(\sigma)$ in the sample, combined with a number of geometrical loss factors. For most situations in magnetic data storage research, the films under investigation are thin and film thickness loss term $\left(1-e^{-|\boldsymbol{k}| t}\right)$ will dominate over the tip height term $\left(1-e^{-|\boldsymbol{k}| h}\right)$. If we further assume that the tip cross-section $(b \times S)$ is much smaller than the smallest features of the charge distribution in the film, we get a very simple expression for the TTF

$$
\widehat{F}_{z}(\boldsymbol{k}, z)=-\mu_{0} M_{t} b S\left(1-e^{-|\boldsymbol{k}| t}\right) e^{-|\boldsymbol{k}| z} \widehat{\sigma}_{e f f}(\boldsymbol{k})
$$

This approximation can be called the monopole approximation, because we assume that all magnetic charges $\left(M_{t} b S\right)$ are located at one point at the end of the tip, and that the other charges are very far away from the sample surface. The only loss terms that remain are the film thickness loss, and the tip-sample distance loss, which usually is dominant. This immediately shows that for a good signal to noise ratio (SNR) in the image, the tip/sample distance has to be as small as possible.

When the details in the image start to approach the tip dimensions, the sinc functions have to be considered as well. For this bar type tip, the force becomes zero when the wavelength of the surface charge distribution equals the tip size. This is analogue to the situation in magnetic recording, where at the gap zero the bitsize is half the gap-size of the recording head.

The force we calculate in (20) is measured by means of a cantilever deflection or change in resonance frequency. For the latter case $\partial F_{z} / \partial z$ has to be calculated, which in the Fourier domain is simply:

$$
\frac{\partial \widehat{F}_{z}(\boldsymbol{k}, z)}{z}=-|\boldsymbol{k}| \widehat{F}_{z}(\boldsymbol{k}, z)
$$

A typical example of TTFs for the static and dynamic mode and the resulting deflection and resonance shift, calculated from (1) and (12), is given in figure 5 , using the parameters from table 1.

\subsection{Instrumentation}

The scope of this book does not validate an exhaustive discussion of scanning probe microscope instrumentation. When using MFM or making decisions on the type of instrument to be used, some background information can be usefull. For an excellent example of modern microscope design see $\mathrm{Hug}$ et al.[13]. In scanning probe instrumentation we can recognize the following subsystems: 

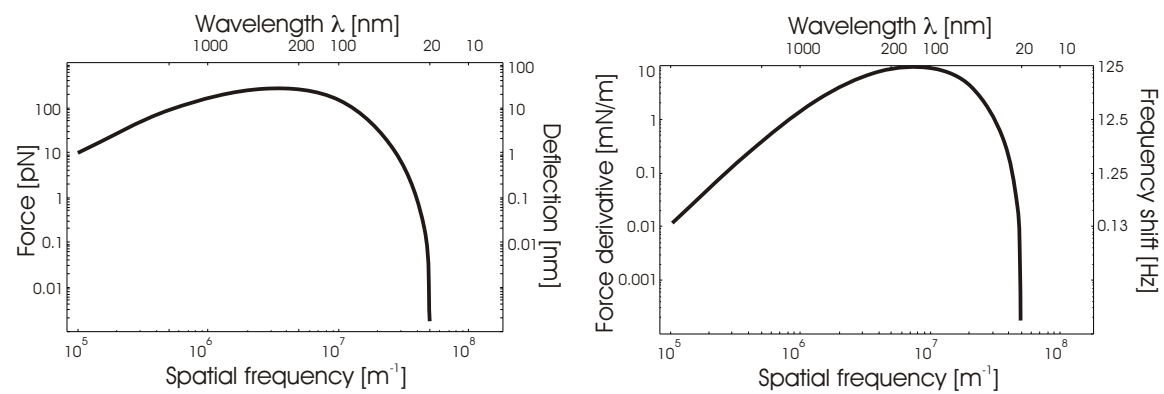

Fig. 5. Tip transfer functions for a typical situation (table 1)

$\begin{array}{ll}M_{t} & \text { Tip magnetization } \\ b & \text { Tip thickness (coating thickness) } \\ s & \text { Tip width } \\ h & \text { Tip length } \\ M_{s} & \text { Sample saturation magnetization } \\ t & \text { Sample thickness } \\ z_{0} & \text { Tip sample distance } \\ c & \text { Cantilever spring constant } \\ f_{n} & \text { Cantilever resonance frequency }\end{array}$

$1422 \mathrm{kA} / \mathrm{m}$
$20 \mathrm{~nm}$
$100 \mathrm{~nm}$
$1 \mu \mathrm{m}$
$295 \mathrm{kA} / \mathrm{m}$
$70 \mathrm{~nm}$
$20 \mathrm{~nm}$
$.01(3) \mathrm{N} / \mathrm{m}$
$7(75) \mathrm{kHz}$

Table 1. Parameters used in the calculation of the tip transfer functions and noise levels of figure 5. Values in parentheses are for the dynamic mode curves

Probe The force on the magnetic tip is detected by means of a cantilever and a displacement sensor. The tip and cantilever will be discussed in detail in section 5 , because the tip size obviously determines the ultimate resolution of the MFM. But this resolution can only be obtained if the detection system is capable of measuring the very small deflections of the cantilever. Currently only optical displacement sensors are used in high resolution MFM, such as beam deflection systems or various types of interferometers. The beam deflection system is very robust and easy to align, but calibration of the sensor is only possible by indirect methods, such as calibration grids. The interferometer on the other hand is more difficult to align, but can be easily calibrated with respect to the laser wavelength. By using a fiber interferometer [25], the active size of the interferometer can be made extremely small, which is beneficial to the instrument stability. Whether the beam deflection or interferometer detection system is more sensitive depends very much on the implementation, but in principle the interferometer is a factor of 4 more sensitive[7]. The detection limits is estimated to be as low as $10^{-15} \mathrm{~m} / \sqrt{\mathrm{Hz}}[30]$.

Positioning The positioning system brings the sample close to the tip (coarse-positioning) and scans the tip over the sample area to obtain an im- 
age (fine-positioning). The coarse positioning system has the difficult task to achieve $m m$ displacements with a stability better than $1 \mathrm{~nm}$ (when switched off). Ordinary manual or moterized screw postioners are used very often, sometimes in combination with levers. These systems suffer from drift: it can sometimes take hours before the displacement relaxes. Hysteresis is also a big problem. For vacuum systems (see next) these screw positioners are not really usefull since they require greasing, allthough some vacuum compatible systems do exist. Therefore recent microscope designs resort to the use of piezo-actuated stepper actuators. Principles such as slip-stick, inertia and walking -beetle- motions are used. The big advantage of these systems is that they are extremely stable once switched off. The disadvantage is that they are much more difficult to operate and sometimes do not move at all, which is a nightmare for instrument designers: you have a perfect MFM and you cannot get the tip close to the sample...

The fine positioning system has the difficult task to achieve an as big as possible scanrange with sub- $n m$ stability, and on top of that scan as fast as possible. For speed and stability it is advantageous to use a small scanner, but of course this is contradictory to the scan-range requirement. In most microscopes so called tube-scanners are used. The major disadvantage of the tube scanner is the coupling between the $x y$ - and $z$ motion because the sample is tilted as well as translated. A very nice tutorial on piezo-translators can be found the website of Physik Instrumente [4].

Housing Although seemingly trivial, the environment in which the MFM instrumentation is mounted is of crucial importance to the image quality. The MFM works at extremely small tip/sample distances of $30 \mathrm{~nm}$ and less, but the tip never touches the sample surface. The mechanical path from tip to sample is therefore several centimeters, six to seven orders of magnitude larger than the tip/sample distance. Therefore the MFM is extremely sensitive to temperature variations, mechanical and acoustic vibrations and air flow. For high resolution the MFM is mounted on a vibration isolation table (figure 6), in an environment which shields acoustic noise and air flow. Air operated instruments are put in noise isolation cupboards, which enclose both the MFM and the vibration isolation table. To elimate electromagnetic noise, the cupboard can be equipped with wire-mesh to provide a Faraday cage. Sometimes ionizing units are mounted inside the cupboard to prevent static charge on the sample.

The ultimate acoustic noise isolation is obtained when the MFM is mounted in vacuum. A vacuum environment has many other important advantages. Damping of the very small cantilever is dominated by impingment of air molecules, which counteracts cantilever movement, and squeezing of air between the cantilever and the sample. The quality factor of resonance $Q$ goes up by several orders of magnitude when the the air is removed. This increase in quality factor strongly increases the instruments sensitivity, and therefore the resolution (see section 3.2). Next to this, Brownian motion of the air 

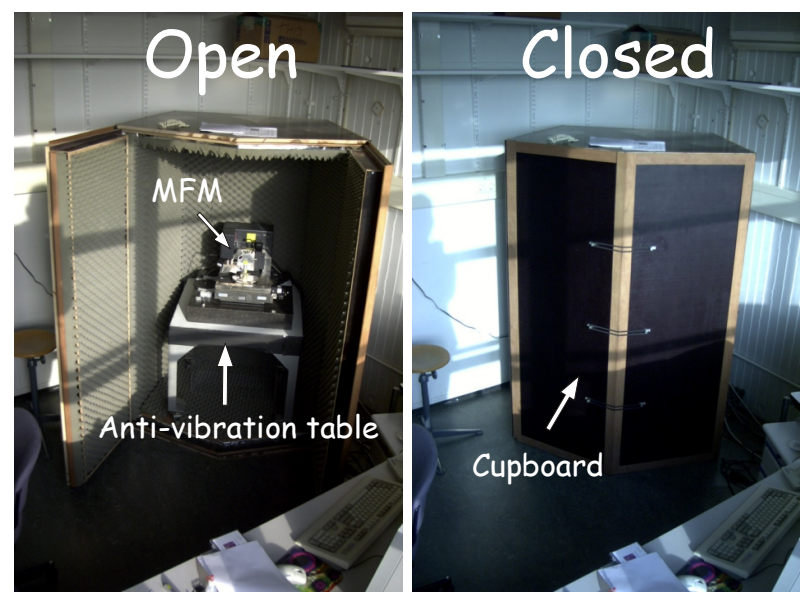

Fig. 6. Noise isolation cupboard in the authors laboratory featuring non-parallel side-walls, two layer sound isolation, a Faraday cage and ionizing unit (not visible)

molecules excite the cantilever as well and cause additional noise. Finally the vacuum removes a large part of the gasses which stick to the sample surface, such as water. Especially the highly mobile molecules on the surface cause a problem, since they move towards the tip and form a meniscus, which pulls the tip into the sample. A vacuum of about $0.1 \mathrm{~Pa}\left(10^{-3} \mathrm{mbar}\right)$ is sufficient to benefit fully from the effect of vacuum. Care must be taken however that the pressure is not too high, the conditions for electric breakdown between the piezo-electrodes are optimal for pressures between $1 \mathrm{~Pa}$ and $40 \mathrm{kPa}\left(10^{-2}\right.$ mbar and 0.4 bar)[19]. Since $1 P a$ is difficult to reach with a rotary pump only, two-stage pumping systems are therefore almost a necessity.

Control The actual acquisition of an image requires a complicated control system, which takes care of tip sample positioning, both for scanning and for controlling the tip-sample distance, data acquisition and visualisation and possibly other control loops such as phase locking in dynamic mode measurements. With the gradual process in Digital Signal Processing (DSP), more and more tasks are being taken over by digital control systems. (This is how one of the biggest scanning probe microscope manufacturers got its name). Still high quality analog electronics, such as the pre-amplifiers and high voltage amplifiers for the piezo's, are crucial and should not be neglected.

In the design of control electronics two themes play an important role. First of all the noise caused by the control systems should be minimimal. This means that high quality components, such as powerful DSPs and low noise, high bandwidth DA converters, have to be used. As a result, for microscopes operated in air, the price of the control system usually equals that 
of scanning probe microscope hardware. In the second place the control system should provide a user-friendly interface to the microscope system with a lot of flexibility in scanning modes and parameter tuning. Unfortunately, every single scanning probe microscope manufacturer has his own dedicated front-end, using proprietary code. Until now there have been no attempts to standardize, not even in the format of the output data files. This seriously hampers the transfer of data between different research laboratories, which often use different brands of microscopes. I believe it is the task of the academic community to improve this situation, and propose such a standard.

\section{MFM in Magnetic Data Storage Research}

The MFM has found a widespread application in magnetic recording research, mainly because it is a relatively cheap high resolution imaging technique which does not require sample preparation. Moreover, in magnetic recording one designs media and heads in such a way that the surfaces are very smooth and the external stray fields are very high, both of which are beneficial to MFM. And finally, recording media are very resistant to the influence of external fields, so tip/sample interaction is not really a problem. It is therefore not coincidental that reference samples for MFM originate from the magnetic recording community (such as the NIST hard disk[23] or the Magneto-optic disk used for the CAMST reference sample[1]).

The magnetic recording industry is however on an incredibly steep roadmap. Each year the data density is more than doubled. Progress in MFM technology has been made mainly on the ease of use of the instrumentation. In the early nineties an MFM measurement could take a week. The author painfully remembers that it could take a day to prepare an MFM tip, but a second to break it. Much has done by the microscope industry to improve upon this situation. The use of batch fabricated tips, auto-approach and digital signal processing has brought the total time from sample mount to the first image down to ten minutes. The resolution has however not improved dramatically. Figure 7 shows a more or less representative selection of MFM resolution since its invention. Already in 1991 a resolution as low as $40 \mathrm{~nm}$ was obtained[8], and it took ten years to bring this down to $25 \mathrm{~nm}$. In the meantime the bitlength in experimental hard disks has decreased from $700 \mathrm{~nm}$ in 1990 to $33 \mathrm{~nm}$ in 2001. With the instrumentation and tips we have today, it is very difficult to measure these small bits, and very soon the use of MFM in magnetic recording research will become limited.

Unfortunately (or fortunately, depends on which side you are), there is no reasonable alternative for MFM in magnetic recording research. Electron microscope techniques (such as Lorentz microscopy) require elaborate sample preparation and are not really suitable for perpendicular recording media. Spin polarized STM measurements require extremely clean surfaces, which can up to now only be achieved by in-situ preparation. A very interesting 
question therefore is what the ultimate limit of MFM resolution is. Can we do much better than the 20-30 $\mathrm{nm}$ of today, and what will be needed to achieve that. This is discussed in the remainder of this chapter.

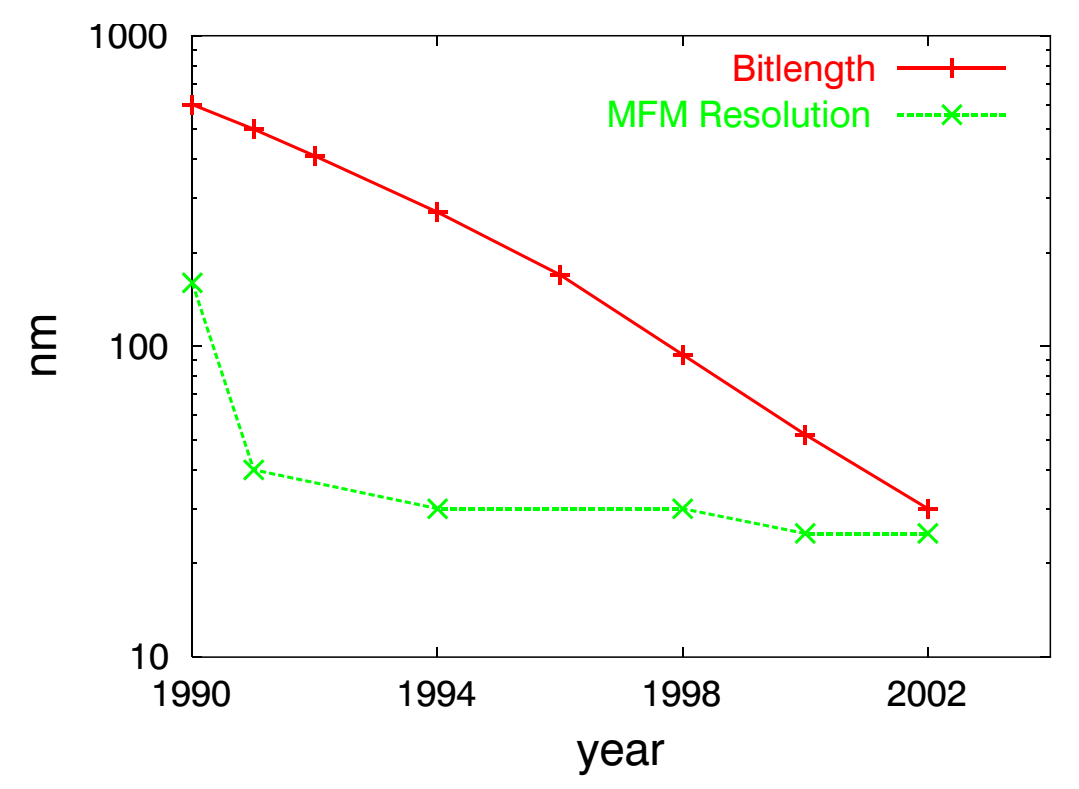

Fig. 7. The progress of magnetic recording has been much faster than the progress in MFM resolution, which might cause a problem in the near future

\section{Limits of resolution in MFM}

The resolution that can be achieved by MFM is determined by the combination of many factors, of which the tip geometry, tip/sample distance and instrument sensitivity are the most important. In this paragraph we set out on a dangerous, but important, task: to determine the ultimate resolution that can be achieved by MFM.

\subsection{Critical wavelength}

Although everyone knows what a high resolution image is, to quantify resolution is not trivial. When an imaging system has high resolution, we usually mean that it is able to separate two closely spaced objects. We do not mean that it can detect one single small object: this has to do with sensitivity. We can define resolution as the minumum spacing between two objects that can still be observed. Instead of two, we can of course take a number of equally 
closed spaced objects. If we take an array, we can even define resolution in a certain direction. By using the array, the definition of resolution can be quite naturally be transferred to the spatial frequency domain as the minimum spacial wavelength that can still be observed. Using the theory of section 1.2, we see that the signal strongly decreases for high frequency components, or small wavelengths. Analog to magnetic recording theory, we can define a certain upper limit on the spatial frequency, above which we call the signal 'nondetectable'. This is usually done with respect to the background noise level: the signal to noise ratio (SNR) should exceed a certain value. For magnetic recording, the SNR should be about a factor of $10(20 \mathrm{~dB})$, for imaging we consider an image still acceptable at an SNR as low as 2 (3 dB) (figure 8).

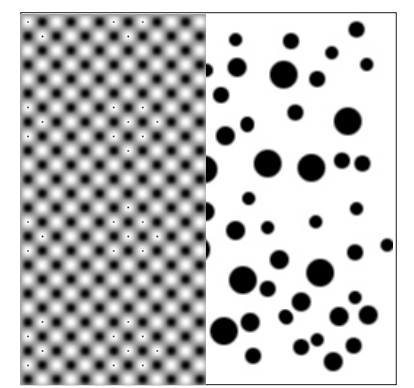

Original image

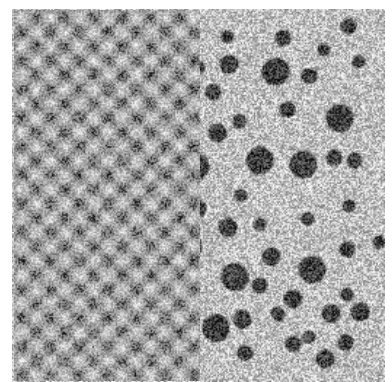

$S N R=2$

Fig. 8. Image quality can be quite acceptable at low signal to noise ratio

In principle the SNR can be made arbitrarily high by increasing the measurement time, because that decreases the measurement bandwidth. This however assumes that the background noise is white, i.e. it has a flat frequency spectrum. In practice, the noise strongly increase when the frequency becomes very low. This is usually referred to as $1 / f$ noise and is caused by drift, for instance by temperature variations or piezo creep. As an illustration, let us take the schematic noise spectrum of figure 9 . The measurement bandwidth $\Delta B$ is related to the measurement time between the pixels $\Delta t$ and the total number of pixels $N$, the combination of which determines the total time to measure an image.

$$
\Delta B=\frac{1}{\Delta t}-\frac{1}{N \Delta t} \approx \frac{1}{\Delta t}
$$

We see that the bandwidth is inversely proportional to the measurement time. But at long measurement times, the bottom of the measurement band will enter the $1 / f$ noise region, which is indicated by $\Delta B^{\prime}$ in figure 9 . So if we exceed a certain measurement time, the total noise (there area under the 
curve) will start to increase, and cancel out the effect of a smaller bandwidth. In MFM, this situation results in a 'stripy' image, where the individual scanlines have noticeable offsets with respect to each other. Image processing can partly eliminate this effect (remove line average), but only if the low frequency noise periods are much longer than the time between scanlines. (One should be careful however, purists will consider this type of image processing scientific fraud). So for reasons of image quality, and of course for practical reasons, we always have to take a limited measurement time into account. A minumum bandwidth of $200 \mathrm{~Hz}$ is reasonable. This allows us to link noise in the frequency domain to noise in the spatial domain through the scanspeed. Now we can find the resolution in figures like figure 10, by calculating the wavelength where the signal drops below the noise level multiplied with the desired SNR. Because the minumum SNR is quite low and usually the tip transfer function is very steep around this point, we simply set the SNR to unity. We call this point the critical wavelength or $\lambda_{c}$.

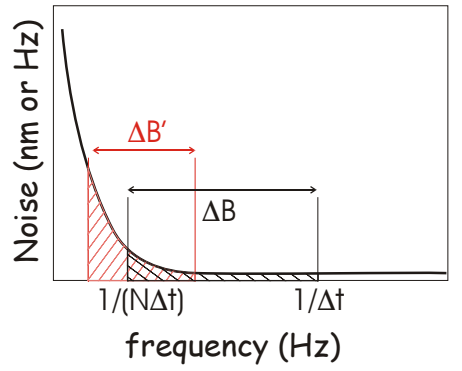

Fig. 9. Increasing the measurement time can increase low frequency noise

\subsection{Thermal noise limited resolution}

Using our simple bar type tip model and the critical wavelength, it must be possible to predict the resolution of MFM, if we know the background noise level. Noise is of course originating from many sources: thermal agitation of the cantilever, electronic cross-talk, mechanical vibration etc. Many noise sources can be eliminated by proper design of the detection system and operation in vacuum. In the ideal situation, we are only left with the thermal noise in the cantilever. This provides us with a kind of fundamental limit to MFM resolution. Whether or not MFM will ultimately reach this limit depends on many factors, but it can serve as a benchmark to compare progress against. 


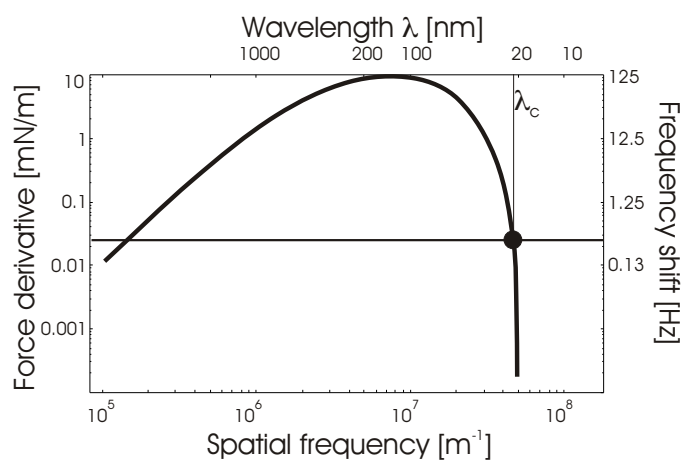

Fig. 10. Using the background noise level, we can define the limit of resolution by means of a critical wavelength $\left(\lambda_{c}\right)$

\subsubsection{Thermal Noise}

The atoms within the cantilever vibrate around an equilibrium position and exert small forces on the cantilever. On the average these forces average out, but at the small scales where we measure in MFM, we can actually observe small fluctuation in the cantilever position with time. When we assume that the cantilever has only one free degree of motion, one can prove that the total thermal energy in the system is $\frac{1}{2} k T$. This energy is equivalent to the average mechanical energy. So we can express the average thermal energy $\left\langle U_{t h}\right\rangle$ in the average amplitude of vibriation of the cantilever end $\left\langle\Delta z_{t h}\right\rangle$, through the spring constant $c$ :

$$
\begin{gathered}
\left\langle U_{t h}\right\rangle=\frac{1}{2} k T=\frac{1}{2} c\left\langle\Delta z^{2}\right\rangle \\
\operatorname{with}\left\langle\Delta z^{2}\right\rangle=\frac{1}{T} \int_{0}^{T}(\Delta z(t))^{2} d t=\left(\Delta z_{r m s}\right)^{2}\left[m^{2}\right]
\end{gathered}
$$

The time average deflection of the cantilever is of course an interesting value, but it does not give us the required noise frequency spectrum. For this we move into the frequency domain using Fourier analysis.

$$
\begin{array}{r}
\widehat{z}(\omega)=\frac{1}{2 \pi} \int_{-\infty}^{\infty} z(t) e^{-i \omega t} d t \\
z(t)=\int_{-\infty}^{\infty} \widehat{z}(\omega) e^{i \omega t} d \omega
\end{array}
$$

The mean square of the displacement translated to the Fourier description becomes 


$$
\left\langle z^{2}\right\rangle=\int_{-\infty}^{+\infty} \widehat{z}(\omega)^{2} d \omega\left[m^{2}\right]
$$

Like in equation (9), we can now calculate the cantilever displacement from the force using the cantilever transfer function:

$$
\begin{array}{r}
\widehat{z}(\omega)=G(\omega) \widehat{F}(\omega)[m / H z] \\
G(\omega)=\frac{1 / m}{\sqrt{\left(\omega_{n}^{2}-\omega^{2}\right)^{2}+\left(\omega \omega_{n} / Q\right)^{2}}}[m / N]
\end{array}
$$

We assume that force the range of frequencies we are interested in, the 'thermal force' of the combined action of all atoms is equally distributed over all frequency components (so $\widehat{F}(\omega)=\widehat{F}_{t h}$ ). This 'thermal force' is usually called the thermal white noise drive. The cantilever vibration spectrum therefore simply equals $G \widehat{F}_{t h}$. In a cantilever with a high quality factor, the noise will predominantly focus around the resonance frequency and the noise at $\mathrm{DC}$ will be low.

In the Fourier domain, we can relate the mechanical energy to the thermal white noise drive:

$$
\widehat{U}_{t h}(\omega)=\frac{1}{2} c \widehat{z}_{t h}^{2}=\frac{1}{2} c G^{2} \widehat{F}_{t h}^{2}
$$

Integrating over all spectral components yields the thermal energy, which was equal to $\frac{1}{2} k T$, so we can calculate the frequency independent thermal white noise drive:

$$
\begin{array}{r}
\left\langle U_{t h}\right\rangle=\int_{-\infty}^{\infty} \widehat{U}_{t h}(\omega) d \omega=\frac{\left(\Delta F_{t h}\right)^{2} Q \pi \omega_{n}}{2 c}=\frac{1}{2} k T \quad[J] \\
\rightarrow \Delta F_{t h}=\sqrt{\frac{k T c}{\pi \omega_{n} Q}}=\sqrt{\frac{k T m \omega_{n}}{\pi Q}}=\sqrt{\frac{k T D}{\pi}}[N \sqrt{r a d / s}]
\end{array}
$$

The thermal white noise drive results in a frequency dependent cantilever movement, which can now simply be calculated from the transfer function $\Delta z_{t h}(\omega)=G(\omega) \Delta F_{t h}$. For static mode MFM, the bandwidth is usually far below the cantilever resonance frequency and we can approximate the thermal noise to $\Delta z_{t h}(\omega)=\Delta F_{t h} / c$

The average noise in a measurement bandwidth from 0 to $\Delta \omega$ equals 


$$
\begin{gathered}
\left\langle\Delta F_{t h}^{2}\right\rangle=\int_{-\Delta \omega}^{\Delta \omega} \Delta F_{t h}(\omega) d \omega=\frac{2 k T c \Delta \omega}{\pi \omega_{n} Q}\left[N^{2}\right] \\
\left(\Delta F_{t h}\right)_{r m s}=\sqrt{\frac{4 k T c \Delta B}{\omega_{n} Q}}=\sqrt{k T D \Delta B}[N]
\end{gathered}
$$

With $\Delta B=2 \pi \Delta \omega[H z]$. In the last expression we can appreciate the analogy with the thermal white voltage noise (Johnson noise) in an electric resistance $\sqrt{4 k T R \Delta B}[V]$.

For dynamic mode MFM, we have to analyse the noise in a self-oscillilating system with positive feedback. This has been done by Albrecht[2], who obtained

$$
\left(\Delta\left(\frac{\partial F}{\partial z}\right)_{t h}\right)_{r m s}=\sqrt{\frac{4 k T c \Delta B}{\omega_{n} Q\left\langle z_{o s c}^{2}\right\rangle}}
$$

Where $\left\langle z_{\text {osc }}^{2}\right\rangle$ is the mean square amplitude of the self oscillating cantilever, to which the minimum detectable force derivative is inversely proportional.

\subsubsection{Critical wavelength}

Using equations (34), (35)) for the noise levels we can now calculate the critical wavelength for the bar tip model (equation (20)). To obtain simple expressions, we set the bar tip cross section square so that we have the same resolution in $x$ and $y$ direction and we restrict ourselves to the $x$-direction. Furthermore we assume that the tip height $h$ is much larger than the film thickness $t$, and that the film thickness loss factor can be ignored for wavelengths close to the critical wavelength. These assumption are not so strict, but to reduce the number of parameters even further we also assume that the tip/sample distance loss $\left(e^{-k_{x} z}\right)$ can be ignored. This is approximately true for $z<0.1 / k_{x}$, or $z<0.02 \lambda_{c}$. To achieve such small tip/sample distances is not trivial, and we come back to that later. Doing so however, we obtain a fairly simple expression for the force:

$$
\widehat{F}_{z}(\boldsymbol{k}, z)=-\mu_{0} M_{t} b^{2} \operatorname{sinc}\left(\frac{k_{x} b}{2}\right) \operatorname{sinc}\left(\frac{k_{y} b}{2}\right) \widehat{\sigma}(\boldsymbol{k})\left[N m^{2}\right]
$$

To calculate the critical wavelength, we assume a perpendicular medium with a harmonic magnetization distribution with identical wavelength in $x$ and $y$ direction: $M_{z} \sin (2 \pi x / \lambda) \sin (2 \pi y / \lambda)$ (see figure 8 for instance). We than obtain for the force amplitude simply 


$$
\Delta F_{z}(\lambda)=\mu_{0} M_{t} M_{z} b^{2} \operatorname{sinc}^{2}\left(\frac{\pi b}{\lambda}\right)[N]
$$

For a fixed wavelength $\lambda$, the force has a maximum amplitude at $b=\lambda / 2$, so when the tip diameter is exactly equal to half the wavelength of the stray field. This is logical, because for smaller wavelengths charges with opposite polarity will be beneath the tip and reduce the force. The tip dimension $b$ can now be eliminated, leaving

$$
\left(\Delta F_{z}(\lambda)\right)_{\max }=\mu_{0} M_{t} M_{z}\left(\frac{\lambda}{\pi}\right)^{2}[N]
$$

This force should be larger than the base noise level we obtain for the critical wavelength

$$
\lambda_{c}=\pi \sqrt{\frac{\sqrt{4 k T D \Delta B}}{\mu_{0} M_{t} M_{s}}}
$$

With equation (39), we have a lower fundamental limit to the resolution of MFM, in other words this is the resolution which is theoretically possible. Let us take static mode MFM in air at room temperature. With $c=0.03 \mathrm{~N} / \mathrm{m}$, $\omega_{n}=2 \pi 10 \mathrm{kHz}, Q=30$ and a bandwidth $\Delta B$ of $200 \mathrm{~Hz}$ we get a thermal noise level of about $1.5 \mathrm{pN}$. Taking both $M_{s}$ and $M_{t}$ equal to $1 \mathrm{MAm}^{-1}$, we get a critical wavelength of $3.4 \mathrm{~nm}$, which means feature sizes of $1.7 \mathrm{~nm}$. This extremely low value is achieved with a tip of $1.7 \mathrm{~nm}$ in diameter and at a tip/sample distance of less than $0.07 \mathrm{~nm}$. Of course this tip/sample is impossible to achieve in static mode MFM. Our first problem will be that we have contamination layers on the tip and sample. Suppose that we solve this by moving to UHV and in-situ preparation of tip and sample, than at this distance we will have strong VanderWaals forces, so that the tip will be pulled into the sample if the spring constant is too low. Furthermore we assumed in our calculations that the magnetic charges are at the tip and and sample surface. Of course they are not, we expect the charges to be distributed over the exchange length, which is a few $\mathrm{nm}$ in general. What we can conclude however, is that even for the simple case of static mode MFM in air, we can do much better than the $30 \mathrm{~nm}$ resolution ( $60 \mathrm{~nm}$ critical wavelength) which is observed today.

So let us be realistic, and assume that we can achieve a tip/sample distance $z_{\min }$ of at least $10 \mathrm{~nm}$. The signal is now reduced by a factor $\exp \left(-2 \pi z_{\min } / \lambda\right)$ and

$$
\left(\Delta F_{z}(\lambda)\right)_{\max }=\mu_{0} M_{t} M_{z} e^{-2 \pi z_{\min } / \lambda}\left(\frac{\lambda}{\pi}\right)^{2}[N]
$$


which is kind of akward to solve analytically for $\lambda$. Numerically we obtain figure 11, which shows that we can achieve resolution better than $20 \mathrm{~nm}$ in air (10 $\mathrm{nm}$ feature size). If we move to vacuum and increase the quality factor to $10^{4}$ for instance, the noise level decreases to $0.08 \mathrm{pN}$ and the critical wavelength is smaller than $12 \mathrm{~nm}$, with minumum of $0.8 \mathrm{~nm}$.

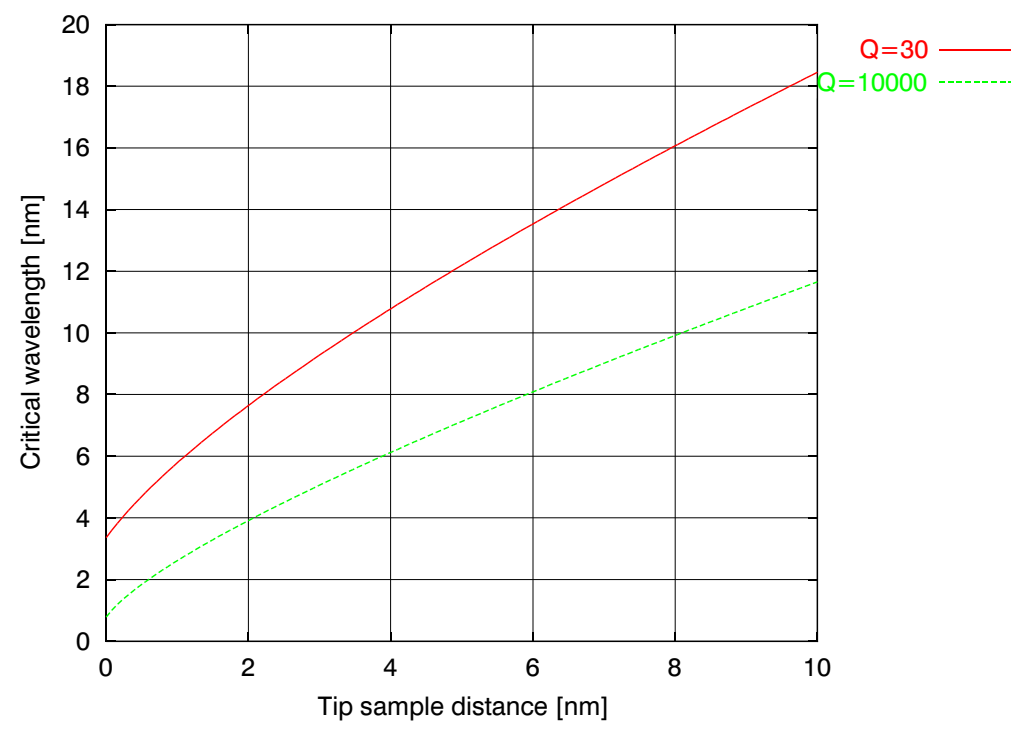

Fig. 11. Thermal noise limited critical wavelength for static mode MFM in air $(\mathrm{Q}=30)$ and vacuum $(\mathrm{Q}=10000)$, using $c=0.03 \mathrm{~N} / \mathrm{m}, \omega_{n}=2 \pi 10 \mathrm{kHz}$ and a bandwidth $\Delta B$ of $200 \mathrm{~Hz}$

We can perform the same exercise for dynamic mode MFM, using the thermal noise background of equation (35). The oscillation amplitude is another parameter, which is set equal to its maximum value of the tip/sample distance $z_{\min }$ (so it almost hitting the sample). In principle we cannot use the theory of section 1.1 for dynamic mode MFM in this case, because the force derivative will not be constant over the cantilever oscillation. But let us regard the calculated (wrong) values as a upper limit, than if we take for example a typical MFM cantilever with $\omega_{n}=2 \pi 75 \mathrm{kHz}$ and a spring constant $c=3 \mathrm{~N} / \mathrm{m}$ we obtain the curves of figure 12 in $\operatorname{air}(Q=300)$ and vacuum $(Q=40000)$. The deterioration of resolution for very small tip/sample distance is caused by the fact that the noise levels increase faster than the signal. In fact for very small distances static mode MFM has a lower theoretical resolution than dynamic mode MFM. For larger tip sample distances, the dynamic mode is better, allthough the differences are not dramatic. In general we can conclude that 
when we move to vacuum, the theoretically achievable resolution of MFM is improved by a factor of two.

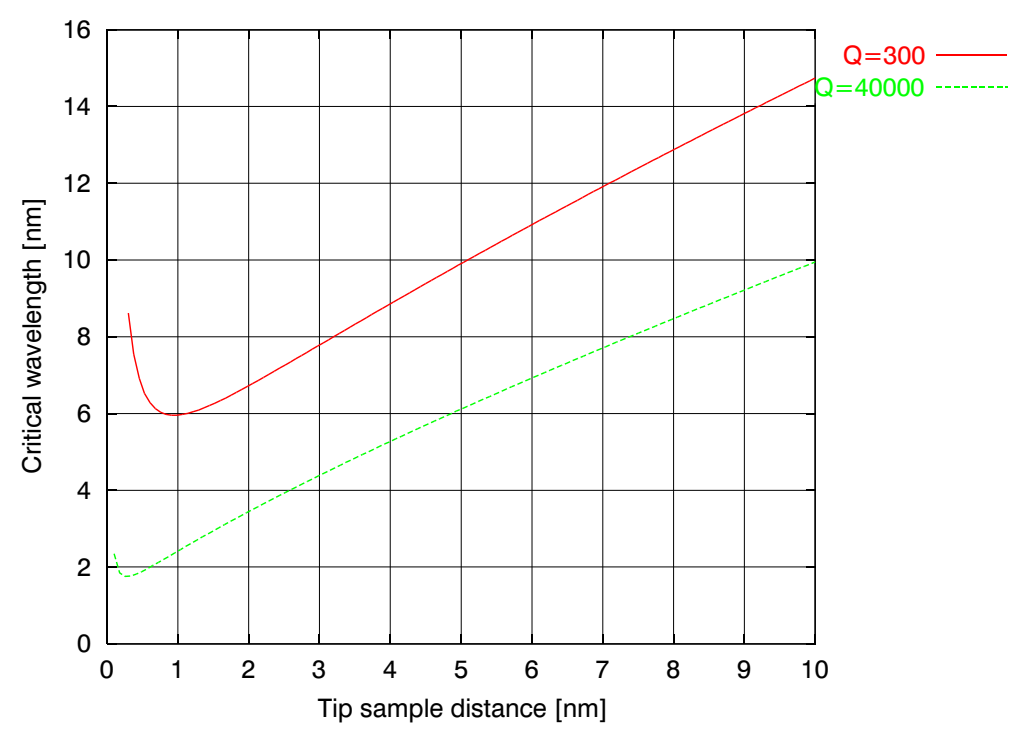

Fig. 12. Thermal noise limited critical wavelength for dynamic mode MFM in air $(\mathrm{Q}=300)$ and vacuum $(\mathrm{Q}=40000)$, using $c=3 \mathrm{~N} / \mathrm{m}, \omega_{n}=2 \pi 75 \mathrm{kHz}$ and a bandwidth $\Delta B$ of $200 \mathrm{~Hz}$

\subsubsection{Beware!}

Allthough the above analysis of $\lambda_{c}$ is usefull in a sense, its meaning for practical MFM is limited. We emphasise again that the analysis above gives a theoretical upper limit to the resolution, under the condition that the only noise source is thermal noise in the cantilever and that the imaging process can be modelled by the bar tip model. Of course there are other noise sources, such as laser power fluctuation in case of beam deflection or interferometry, $1 / f$ noise in the system caused by temperature drift and cross talk from high voltage positioning signals for piezos and external sources. Even in the quality factor, which determines the thermal noise level, there is an uncertainly. Damping caused by air squeezing between the cantilever and the sample, meniscus formation and eddy currents in the sample caused by the time varying magnetic stray field of the tip will reduce the quality factor if the tip is approached to the sample. It is therefore wise to measure the quality factor of resonance at the same tip/sample distance as used during the measurement. 
In our calculations we used on one hand the thermal noise, which gives a lower limit on the noise level. On the other hand we used the idealized bar tip model, which gives an upper limit to the signal level. Usually we find however much smaller signals. There are many possible reasons for this. One reason might be that the magnetic charge density will not be on the uttermost front surface of the tip, nor at the top surface of the medium. It is likely that the charges will be distributed over a volume with a thickness of at least the exchange length, which can be several nanometers. Fortunately the area over which the charges are distributed decreases with the tip dimension. Figure 13 shows the result of two micromagnetic calculations on tips of different sizes (using MagFEM3D, a large scale micromagnetic calculation package [22]). The magnetisation in the large tip $(20 \times 100 \mathrm{~nm})$ is able to rotate away from the front surface, with the result that only $50 \%$ of the charges are on the tip front surface. In the much smaller tip $(7 \times 20 \mathrm{~nm}) 98 \%$ of the charges are on the front surface, simply because there is no more space for the magnetisation to rotate away.
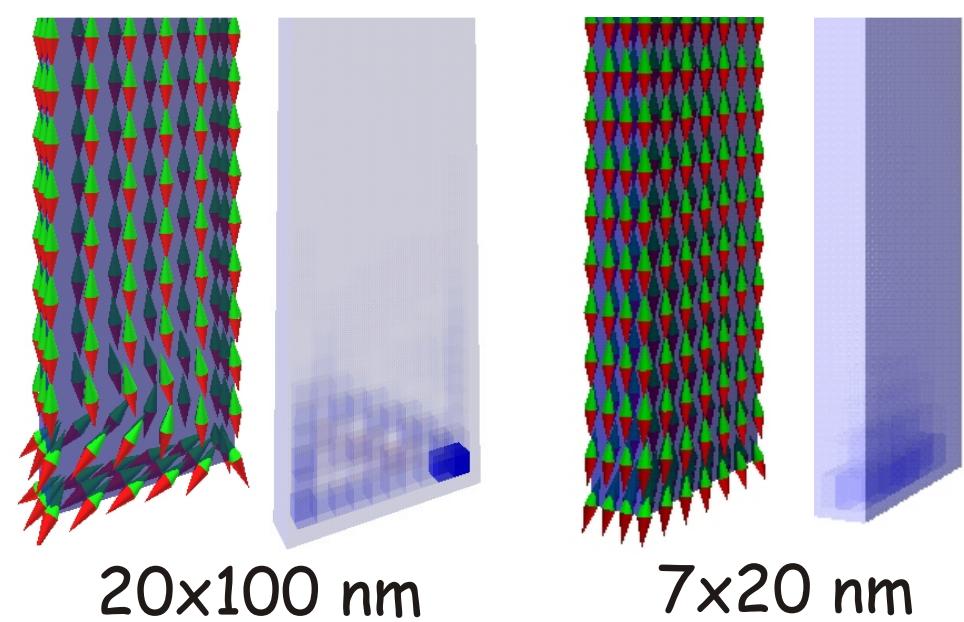

Fig. 13. Micromagnetic simulation of equilibrium magnetisation in a bary type tip with a dimension of 20x100 $\mathrm{nm}$ (left) and $7 \times 20 \mathrm{~nm}$ (right). The images show the magnetisation vectors and the volume charge distribution

There are also other reasons besides the charge distribution which create a difference between the magnetic separation and physical separation of the tip and the sample surface. Unless carefully prepared and operated in UHV, the sample surface will have a surface surfactant layer of absorbed molecules. Also the magnetic tip will have such a layer, and the magnetic tip might not extend completely to the end of the tip, for instance because of an oxide layer. All 
these effects result in an effective tip/sample distance which is larger than the physical tip/sample distance. Therefore we usually find a considerably lower signal than predicted by the bar tip model. For the CAMST reference sample for instance [1], we typically find signals in the order of 1-3 $\mathrm{Hz}$, whereas the bar tip model predicts $10-100 \mathrm{~Hz}$.

In conclusion we see that in reality the noise level will be higher, and the signal level lower that the values assumed in the theory outlined above. The limit on resolution presented here can however be used to benchmark progress against. The theory also shows three important areas in which this progress can be made:

- decrease noise

- reduce tip/sample distance.

- improve tip shape and size

The latter two will be discussed in the following.

\section{Tip-sample distance control}

There are at least two reasons why we want to measure and control the tip-sample distance. In the first place we want to be as close as possible to the sample surface to obtain high resolution. Sample tilt, drift in the microscope positioning and surface roughness will cause a variation in tipsample distance over the image, and we can never get closer than the highest point on the surface. In the second place we will get a stronger contribution from non-magnetic forces as we approach closer and closer to the sample, and topographic information will be superimposed on the image. If we keep at constant tip-sample distance, the contribution of those forces will be more or less constant as well, and we obtain a topography-free image.

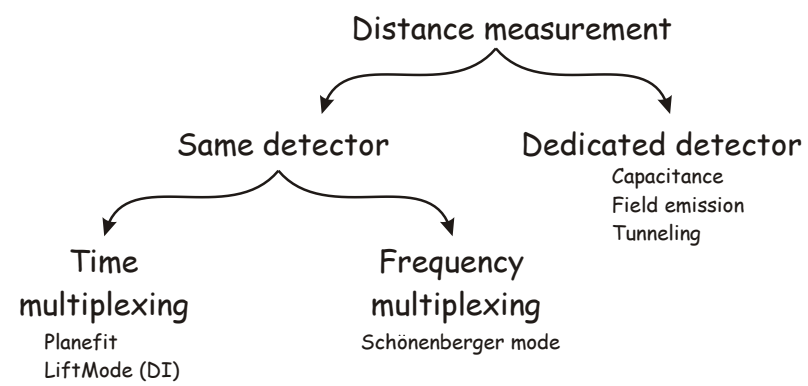

Fig. 14. Different ways to control tip-sample distance

There are various ways to achieve constant tip-sample distance, all differing in complexity, precision and stability. In the following we will try to 
position these approaches in a structure as in figure 14. Probably the ideal situation would be that we use a dedicated detector, optimised for tip-sample distance measurement. STM seems to be a good candidate, because it will enable us to control the tip-sample distance with $\AA$ precision. The disadvantage might be that we only get reasonable tunneling currents at very low tip-sample distances, too low to allow for sufficiently soft cantilevers in static mode MFM or for sufficiently large vibration amplitudes in dynamic mode MFM. In the future STM control might become an option however. At higher tip-sample distances we can still use the STM technique, but than in the field emission mode. This requires higher tip-sample voltages and the resolution will not be as good as in STM. Another option might be to measure the capacitance between the cantilever or tip and the sample.

All these options require complex integrated probes with bond pads and wires running down the cantilever. That is why currently practically all methods to measure the tip-sample distance use the same detector as the one used for measuring the magnetic force (beam deflection, interferometer, piezo-resistive etc.). Since we use the same measurement channel, we have to multiplex the magnetic and topographic signals. Multiplexing can be done in the time domain. This is the most commonly used method because it allows you to use AFM to measure the tip-sample distance. Time multiplexing can be done on various scales:

- Image-per-image An example of this method is the real-time plane fit (such as for instance used in control systems manufactured by RHK), which is often used in static mode MFM. First we measure an AFM image of the surface and determine the slope of the image. Than we add a correction signal to the $z$-piezo and make sure that the AFM image appears flat: the average tip-sample distance is constant. More sophisticated systems use a polynomial fit to the surface in which bowing is taken into account. This is especially useful for large range scans using tube scanners. In principle it is not necessary to measure the complete AFM image, depending on the order of the polynomial just a few strategically placed scanlines are sufficient. A big advantage of this mode is that there is no contact between the tip and the sample during image formation. Especially for soft magnetic samples, in which the domain image is easily disturbed by the MFM tip stray field, this mode has an advantage over the other two time-multiplexing modes discussed hereafter.

In principle the plane-fit method can be extended to measuring a complete AFM image and do the distance control on a pixel per pixel basis, but instrument drift during the time it takes to scan an image has to be less than the distance between the pixels. This type of accuray is only found in low temperature microscopes.

- Line-per-line This method is called LiftMode by Digital Instruments and is used with dynamic mode MFM. Every line in the image is scanned twice. The first scan is in tapping mode AFM with amplitude detection 
(see section 1.1). The traced topography is stored in memory and used to control the tip-sample distance in the subsequent MFM trace, using phase or frequency detection. Since the time per line trace is relatively short this method works quite well. Moreover the topographic and magnetic information are obtained simultaneously, which makes mapping of topographic features onto the MFM image very easy. The method is less suitable for static mode AFM/MFM, because than very soft cantilevers are needed which snap into the sample. To 'unsnap' the cantilever, the $z$ piezo has to be withdrawn over quite a distance (sometimes more than a $\mu \mathrm{m})$. Piezo hysteresis and non-linearity than make it difficult to approach the sample accurately again.

- Pixel-per-pixel This mode can be compared with the spectroscopy mode used in STM. For every pixel we make a force distance measurement $\left(F_{z}\right.$ as function of $\left.z\right)$. From the snap-in or contact point we can calculate the tip-sample distance, and we can make a map of the magnetic force for different tip heights. Of course this mode is very slow and suffers heavily from $1 / f$ noise. The extra information obtained is useless, one can easily calculate the MFM signal at a height $z$ from images at taken at lower height. One might think that by scanning at different tip-sample distances one can determine the volume distribution of the magnetic charges in the sample, but the nature of the magnetic stray field simply prohibits that[33]. This mode however does allow for the smallest tip-sample spacing, especially in static mode MFM. (Some sceptics however just see this 'spectroscopy' mode as a way to circumvent the DI patent on the LiftMode).

The last branch in figure 14 is the reciprocal to time-multiplexing, which is frequency multiplexing. This means that we measure topographic and magnetic information in different frequency bands. To achieve this, we need to modulate the magnetic force or the force responsible for the topographic signal (or both). Contact mode AFM is not possible in this frequency multiplexing mode, because we cannot measure the magnetic forces simultaneously. So we need a non-contact measurement of the topography. Non-contact mode AFM might be a possibility, but has to our knowlegde never been used before. It will be very hard, if not impossible, to modulate the forces measured during AFM (such as VanderWaals forces), so we have to modulate the magnetic force. This can be achieved by applying an external magnetic field which modulates the magnetisation in the tip. The same field would also act on the sample however, and we will probably only be able to measure hard magnetic samples.

Another way to measure the topography in non-contact mode is to use an electric field. This has been demonstrated by Schönenberger et al[28], who applied a modulated voltage between the cantilever and the sample. The frequency of modulation is well below the resonance frequency of the cantilever, so that the cantilever will start vibrating. The amplitude of vibration is a 
measure for the tip-sample distance and is kept constant. This method can be applied to static mode as well as dynamic mode MFM. In principle the method is similar to the measurement of the tip/sample capacity, the electric force and the change in capacity being related by

$$
F_{e l}=\frac{\partial C}{\partial z} V^{2}
$$

\section{$5 \quad$ Tips}

\subsection{Ideal tip shape}

Most scanning probe microscope tips are pointed and very sharp: the STM tip and AFM tips are atomically sharp, most SNOM tips are tapered optic fibres. One might therefore assume that an MFM tip should also have the shape of a sharp needle. Techniques such as STM, AFM and SNOM however measure very short-range effects, whereas MFM measures long range magnetostatic forces. (The same holds for Electic Force Microscopy (EFM) and electrostatic forces). In STM and AFM only the last atom determines the signal, but in MFM a much larger part of the tip takes part in the image formation. Therefore the optimum tip shape is not a sharp needle but a bar or cylinder with a flat front end [21], analogue to a hard disk head for perpendicular magnetic recording.
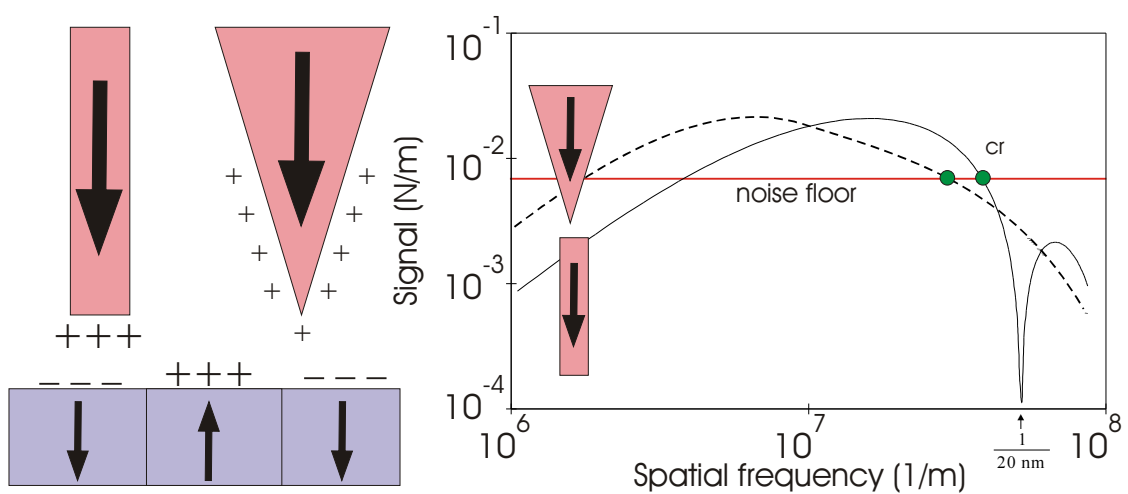

Fig. 15. Point sharp versus bar type MFM tips

The fact that the bar shape is the optimum shape for MFM can perhaps be most easily understood by considering the magnetic charge distribution in a tip with an ideal uniform magnetisation (see figure 15). In a bar type tip all magnetic charges are located at the front surface of the bar, and as close 
as possible to the sample surface. Therefore all magnetic charges contribute equally well to the signal. In a pointed MFM tip, the charge is distributed over the complete tip. The charges located further away from the sample still contribute to the signal, but their effect is stronger at longer wavelengths. If we set the maximum signal of a pointed tip and a bar type tip equal, the bar type tip will perform better at high spatial frequencies, and therefore show a better resolution. Of course, when the wavelengths approach the gap zero of the bar tip, the pointed tip performs better. But we can always tailor the bar type tip in such a way that this happens at wavelengths above the critical wavelength, as indicated in figure 15 on the right. One can argue that we may allow the pointed tip to have higher signal at low spatial frequencies by increasing the tip length. This does however not significantly increase the signal at high spatial frequencies. In all cases the signal close to the critical wavelength will be highest for the bar type tip.

The optimum tip shape is one thing, making such a tip another. When realising MFM tips, we need such small feature sizes that one cannot rely on conventional lithography (as used in semiconductor industry). Instead we have to use tricks to obtain such small dimensions and we enter the area of nanotechnology. The first scanning probe microscope tips were hand-made in a one-by-one fashion. Still the best tips available today are made by hand. The batch fabricated $\mathrm{Si}$ and SiN tips however strongly improved the ease of use the MFM. In the following we will discuss a number of MFM tips, starting with hand made tips, and continuing with batch fabricated tips.

\subsection{Hand made tips}

The first MFM cantilevers were very thin Co or Ni wires which were etched down to a sharp point. The wires were bent around a rasor blade edge, and the laser spot was deflected from the resulting "knee" - a far from stable operation... The tips obtained this way were however not very sharp, and contained a lot of magnetic material[14]. Much better resolution could be obtained with tungsten tips coated with a magnetic layer from the side[24]. The resulting magnetic thin film was however not always smooth, due to the surface roughness on the tungsten point caused by the etching process.

A much smoother tip could be obtained by using contamination needles, which were at that period already used for AFM. These contamination needles were grown in a SEM which was accidently or deliberately contaminated with organic gasses. On the side of these carbon tips, a thin magnetic layer is deposited. Some call these tips Electron Beam Deposited (EBD) tips[5], but to avoid confusion with Electron Beam Evaporation, which is a layer deposition technique, others call these tips Electron Beam Induced Deposited (EBID) tips[26,27]. Especially the tips made by Skidmore et al are impressive, with a tip radius of less than $7 \mathrm{~nm}$. Strange enough, the resolution of the images is not better than obtained with conventional tips[29]. Instead of 
depositing a layer on the side of the $\mathrm{EB}(\mathrm{I}) \mathrm{D}$ tip, one can also use the contamination as an etch protection mask[16]. In this way one can leave a tiny disk of magnetic material at the top of an AFM tip. This MFM tip does not have the ideal shape for high resolution, but can for instance be used in those situations where a low tip switching field is necessary.

The arrival of the Focussed Ion Beam (FIB) instrument opened new possiblities for MFM tip preparation. The two techniques known to the authors start with a magnetic coating on a commercial AFM cantilever. Folks et al [6] start with a commercial MFM tip and FIB etch a small hole at the apex of the tip. The tip is than magnetised in-plane (parallel to the cantilever), so that magnetic charges occur at the edges of the tip. In this way one obtains an in-plane tip which is only sensitive to stray fields parallel to the sample in the direction of the tip magnetisation ( $x$ or $y$ direction). In general one would like to measure the $z$-component, from which the $x$ and $y$ components of the fields can easily be derived. Such a tip is prepared by Philips[17], who starts with a thin Co film on a commercial AFM cantilever, and than etches away the unnecessary material. In this way a $8 \mu \mathrm{m}$ long $50 \mathrm{~nm}$ wide needle could be prepared.
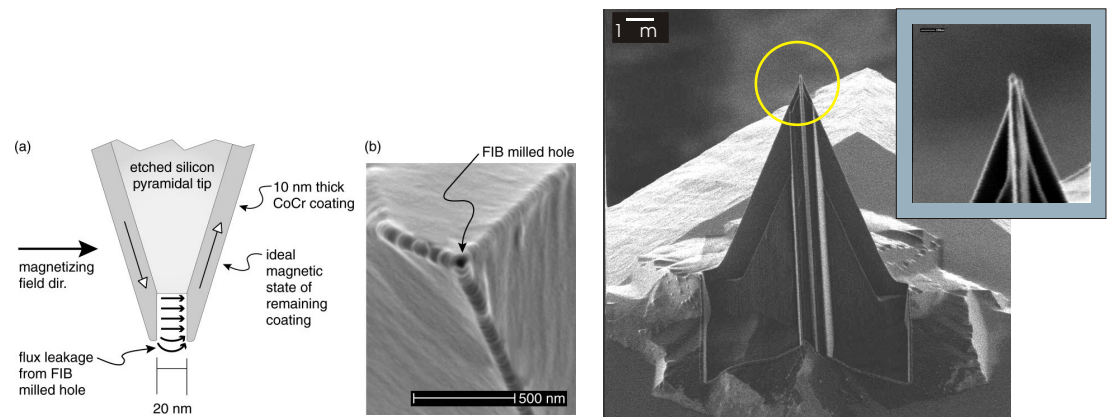

Fig. 16. MFM tips prepared by FIB. Left: Perforated tip [6], right Co needle [18]

\subsection{Coating of AFM tips}

The workhorse of the MFM cantilevers is a Si batch fabricated cantilever with a tip optimized for AFM, which is coated with a magnetic material. There is a large variety in magnetic coatings, and if we try to give an exhaustive list, we would certainly fail. We can however indicate different categories. The most common magnetic coating is an alloy based on Co with additions such as $\mathrm{Cr}$ and $\mathrm{Pt}$, based on the pioneering work of Grütter et al $[9,10]$. These alloys are often derived from hard disk recording layer materials and have a high coercivity. The high coercivity makes the tip resistant against reversal by the 
medium stray field. To achieve a high coercivity, the material is prepared in such a way that one gets magnetically seperated grains. The exact location of these grains on the tip apex varies from tip to tip, which explains why some tips have higher resolution than others. Companies sell tips with different coercivities and film thicknesses (and give them meaningful names such as "high coercivity tip" or "low moment tip").

For some applications, the coercivity of the layers on the tips is not high enough, for instance when measuring stray fields from recording heads or permanent magnets. In that case an alternative can be found in very soft magnetic coatings which switch in very small external fields. As a result however, the tip is always attracted and one looses information on the polarity of the field. Materials used are for instance $\mathrm{Co}[10], \mathrm{NiFe}[10], \mathrm{Fe}[31]$ and granular $\mathrm{Fe}\left(\mathrm{SiO}_{2}\right)$ films[11].

The perfectly soft MFM is a diamagnetic, such as for instance a Pt coated tip. Of course the magnetic moment is much lower than in ferro- or superparamagnetic tips, and the signal is very low[32].

Allthough magnetically coated AFM tips are widely used in MFM, they are a bad approximation to the ideal tip shape. In the following we discuss a new method to realize batch fabricated tip, in a method optimized for MFM.

\subsection{Tip planes: the CantiClever concept}

A magnetic tip that is suitable for high resolution MFM should have lateral dimensions in the nanometer regime. One would like these dimensions to be controllable and variable to be able to customize the MFM tip for different types of measurements or samples. The CantiClever design discussed in this section accomplishes this by defining both lateral dimensions by thin film deposition techniques. The magnetic tip is made by deposition of magnetic material on the side of a free hanging, very thin layer called the tip plane. The width and thickness of the magnetic tip are defined by the thickness of the tip plane and the magnetic layer respectively. The length of the tip is defined using photolithography. A schematic drawing of the structure is shown in figure 17

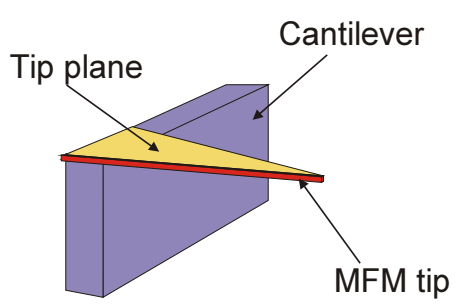

Fig. 17. The cantilever with the tip plane 
Such a structure is very difficult to make however when the approach used to make conventional cantilevers is used. During fabrication, the conventional cantilevers are situated such that the oscillation direction is perpendicular to the surface of the substrate. Using this approach would need the very thin tip plane to be fabricated as a freestanding layer perpendicular to the substrate surface. Instead, the free hanging tip plane is made in a completely new approach. During fabrication, the cantilevers are tilted 90 degrees compared to the conventional cantilevers, creating a lateral oscillating cantilever with its oscillation direction parallel to the substrate surface as shown in figure 18 .

This approach makes the fabrication of the cantilever more difficult compared to conventional cantilevers, but also enables precise control over the cantilever resonance frequency. The resonance frequency is given by [15]:

$$
f_{0}=\frac{1}{2 \pi} C_{0}^{2} \frac{t}{l^{2}} \sqrt{\frac{E_{S i}}{12 \rho_{S i}}}
$$

Where $C_{0}$ is the eigenvalue of the system corresponding to its fundamental frequency, having a value of $1.875, t$ the thickness of the cantilever, $l$ its length, $E_{S i}$ the Young modules of the silicon $(150 \mathrm{GPa})$ and $\rho_{S i}$ its density (2330 $\left.\mathrm{kgm}^{-3}\right)$.

In contrast with the fabrication method used for conventional cantilevers, this approach allows the dimensions of the cantilever which determine the resonance frequency, the cantilever thickness $t$ and the length $l$, to be controlled and varied as both are defined parallel to the substrate surface. A single substrate can carry a large number of cantilevers with different resonance freqencies, suitable for different applications. Furthermore, standard deposition and etching techniques can be used to define the tip plane, as it is also oriented parallel to the substrate surface. The result is a reproducible manufacturing process that incorporates both the cantilever and the magnetic tip and allows for batch fabrication of the probes.

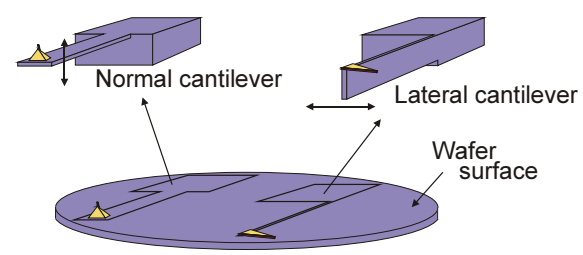

Fig. 18. Tip plane on the cantilever for both cantilever orientations. Left: conventional approach with horizontally vibrating cantilever. Right: new approach with laterally vibrating cantilever 
Two factors play an important role in making a high resolution MFM tip that resembles the ideal tip shape, as described above, as close as possible. First, the free standing tip plane should be very thin, as this defines the width of the tip, but strong enough for contact imaging and at the same time should have low stress to prevent bending. Second, the tip plane should have a well-defined and very sharp cut-off corner as illustrated in figure 19. This ensures that the tip end is flat. How such a sharp cut-off corner is realized, is described in the next section.

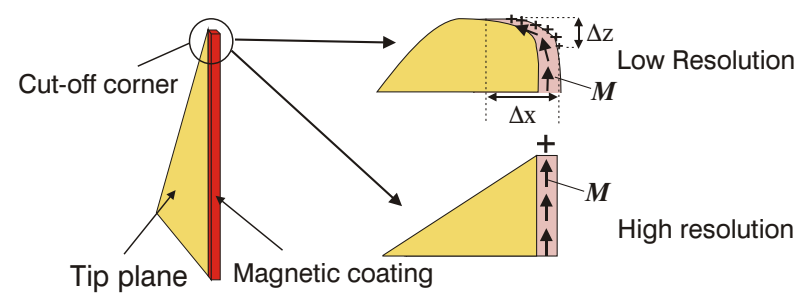

Fig. 19. Influence of the cut-off corner of the tip plane on resolution

The cantilevers are fabricated from (110) silicon wafers using $\mathrm{KOH}$ wet anisotropic etching. The (110) oriented silicon wafers have a $(-1,1,1)$ and a $(1,-1,1)$ plane perpendicular to the wafer surface. The etch rate of the family of 111 planes in $\mathrm{KOH}$ solution is so low that these planes can be considered as etch stop planes. Precise alignment of the cantilevers to these planes will therefore enable lateral cantilevers to be made with a thickness that only depends on the dimensions of the mask because almost no underetching will occur. The crystal plane etch stop also ensures very smooth surfaces, suitable for interferometric deflection detection. The mask material used during $\mathrm{KOH}$ etching are thin silicon nitride layers, deposited in a low stress LPCVD process. The SiN layers are not damaged by the $\mathrm{KOH}$ etching process. This property and the low stress present in the layers make them also perfectly suitable as a material for fabricating the tip planes. The SiN masking layer used during $\mathrm{KOH}$ etching is patterned to form the tip planes. The sharp cut-off corner of the tip plane that is required, is obtained by etching away the blunt tip end at the end of the fabrication process. As a final step, a thin magnetic coating is evaporated on the front side of the tip plane. Precise alignment is important to reduce the amount of magnetic material deposited on the sides of the tip plane.

A SEM photograph of a CantiClever is shown in figure 20. The free hanging tip plane and the smooth sides of the cantilever itself can clearly be distinguished.

Although for this particular probe the tip plane was quite thick (around $100 \mathrm{~nm}$ ) pretty good resolution could be obtained using this batch of tips 


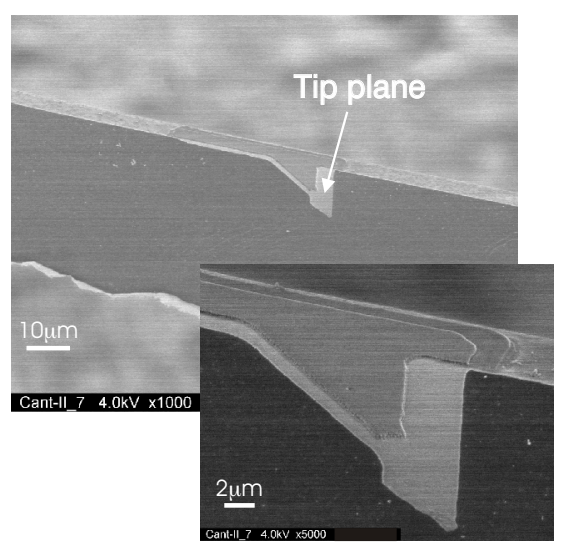

Fig. 20. Cantilever with tip plane and detail of the tip plane (inset)

in MFM. A MFM scan of $0.25 \mu \mathrm{m}$ bits written in a perpendicular harddisk medium is depicted in figure 21 .

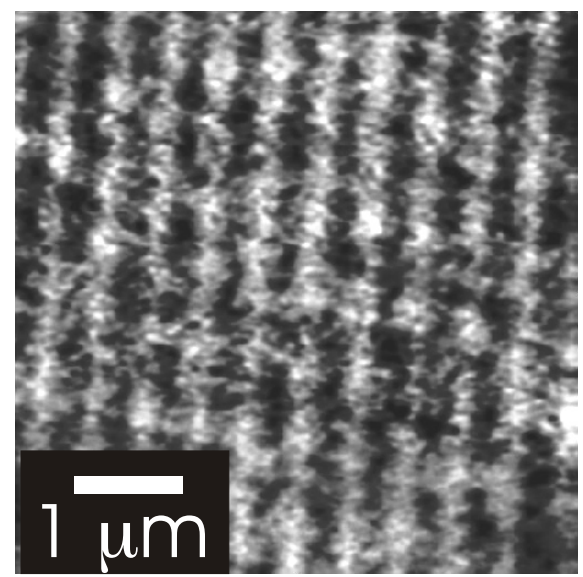

Fig. 21. MFM measurement on an experimental hard disk with $0.25 \mu \mathrm{m}$ bitlength pattern

For this measurement a CantiClever with a $40 \mathrm{kHz}$ resonance frequency was mounted in a commercial MFM from Digital Instruments. The resolution of the tips made using the CantiClever concept can be improved by decreasing the thickness of the supporting tip plane and the magnetic coating. It is expected that the lateral dimensions of the magnetic tip can be reduced below 
$10 \mathrm{~nm}$. Finally, although it is beyond the scope of this text, the CantiClever concept described in this section has also another advantage. Because of the planar nature of the fabrication process, a number of different sensors, besides a MFM tip, can be integrated fairly easily on the tip plane such as a (giant-) magnetoresistive element, making high resolution magnetoresistance microscopy possible.

\section{Acknowledgement}

The authors wish to thank R. Ramaneti for his literature study on MFM tip coatings.

\section{References}

1. L. Abelmann, S. Porthun, M. Haast, C. Lodder, A. Moser, M. E. Best, P. J. A. Vanschendel, B. Stiefel, H. J. Hug, G. P. Heydon, A. Farley, S. R. Hoon, T. Pfaffelhuber, R. Proksch, and K. Babcock. Comparing the resolution of magnetic force microscopes using the camst reference samples. Journal of Magnetism and Magnetic Materials, 190(1-2):135-147, 1998.

2. T. R. Albrecht, P. Grutter, D. Horne, and D. Rugar. Frequency modulation detection using high-q cantilevers for enhanced force microscope sensitivity. $J$ Appl Phys, 69(2):668-673, 1991.

3. D.K. Anand. Introduction to Control Systems. Pergamon Press, Oxford, 2 edition, 1984.

4. Anonymous. Theory and applications of piezo actuators and pzt nanopositioning systems. Technical report, Physik Instrumente, http://www.physikinstrumente.com/tutorial/, 2001.

5. P. B. Fischer, M. S. Wei, and S. Y. Chou. Ultrahigh resolution magnetic force microscope tip fabricated using electron beam lithography. J Vac Sci \& Technol $B, 11(6): 2570-2573,1993$.

6. L. Folks, M. E. Best, P. M. Rice, B. D. Terris, D. Weller, and J. N. Chapman. Perforated tips for high-resolution in-plane magnetic force microscopy. Applied Physics Letters, 76(7):909-911, 2000.

7. A. Garcia-Valenzuela and M. Tabib-Azar. In Massood Tabib-Azar, Dennis L. Polla, and Ka-Kha Wong, editors, Proceedings SPIE, Integrated Optics and Microstructures II, volume 2291, pages 125-142. SPIE, 1994.

8. P. Grütter, H.J. Mamin, and D. Rugar. Magnetic force microscopy. In H.J. Günterrodt and R. Wiesendanger, editors, Scanning Tunneling Microscopy, volume II, pages 151-207. Springer, Berlin, Heidelberg, New York, 1992.

9. P. Grutter, D. Rugar, and H. J. Mamin. Batch faricated sensors for magnetic force microscopy. Appl Phys Lett, 57(17):1820-1822, 1990.

10. P. Grutter, D. Rugar, H. J. Mamin, et al. Magnetic force microscopy with batch-fabricated force sensors. J Appl Phys, 69(8):5883-5885, 1991.

11. P. F. Hopkins, J. Moreland, S. S. Malhotra, and S. H. Liou. Superparamagnetic magnetic force microscopy tips. J Appl Phys, 79(8 Part 2B):6448-6450, 1996.

12. A[lex] Hubert and R[udi] Schäfer. Magnetic domains: the analysis of magnetic microstructures. Springer-Verlag, Berlin, Heidelberg, New-York, 1998. 
13. H. J. Hug, B. Stiefel, P. J. A. Vanschendel, A. Moser, S. Martin, and H. J. Guntherodt. A low temperature ultrahigh vaccum scanning force microscope. Review of Scientific Instruments, 70(9):3625-3640, 1999. Reprint: NOT IN FILE.

14. Y. Martin and H. K. Wickramasinghe. magnetic imaging by 'force microscopy' with 1000 a resolution. Appl Phys Lett, 50(20):1455-1457, 1987.

15. Leonard Meirovitch. Fundamentals of vibrations. Mc Graw-Hill, Boston, 2001.

16. U. Memmert, A. N. Muller, and U. Hartmann. Probes for magnetic force microscopy imaging of soft magnetic samples. Measurement Science $\&$ Technology, 11(9):1342-1347, 2000

17. Gavin Philips, Martin Siekman, Leon Abelmann, and Cock Lodder. High resolution magnetic force microscopy using focused ion beam modified tips. Submitted to Journal of Applied Physics, 2002.

18. G.N. Phillips, L. Abelmann, M. Siekman, and J.C. Lodder. High resolution magnetic force microscopy using focussed ion beam modified tips. Applied Physics Letters, in preparation, 2000.

19. S. Porthun. High Resolution Magnetic Force Microscopy: Instrumentation and Application for Recording Media. PhD thesis, University of Twente, Enschede, The Netherlands, 1996

20. S. Porthun, L. Abelmann, and C. Lodder. Magnetic force microscopy of thin film media for high density magnetic recording. Journal of Magnetims and Magnetic Materials, 182(1-2):238-273, 1998.

21. S. Porthun, L. Abelmann, S. J. L. Vellekoop, J. C. Lodder, and H. J. Hug. Optimization of lateral resolution in magnetic force microscopy. Applied Physics A- Mate Sci Process, 66(Suppl. Part 2):S1185-S1189, 1998

22. K[laus] R. Ramstöck. Magfem3d. http://www.el.utwente.nl/tdm/istg/MagFEM3D/, 1999.

23. P. Rice, S. E. Russek, J. Hoinville, and M. H. Kelley. Optimizing the nist magnetic imaging reference sample. IEEE Trans Magn, 33(5):4065-4067, 1997.

24. D. Rugar, H. J. Mamin, P. Guethner, S. E. Lambert, J. E. Stern, I. McFadyen, and T. Yogi. Magnetic force microscopy: General principles and application to longitudinal recording media. J Appl Phys, 68(3):1169-1183, 1990.

25. D. Rugar, H.J. Mamin, R. Erlandsson, J.E. Stern, and B.D. Terris. Force microscope using a fiber-optic displacement sensor. Review Of Scientific Instruments, 59(11):2337-2340, 1988.

26. M. Ruhrig, S. Porthun, and J. C. Lodder. Magnetic force microscopy using electron-beam fabricated tips. Rev Sci Instrum, 65(10):3224-3228, 1994.

27. M. Ruhrig, S. Porthun, J. C. Lodder, S. McVitie, L. J. Heyderman, A. B. Johnston, and J. N. Chapman. Electron beam fabrication and characterization of high-resolution magnetic force microscopy tips. J Appl Phys, 79(6):29132919, 1996.

28. C. Schonenberger, S. F. Alvarado, S. E. Lambert, and I. L. Saunders. Separation of magnetic and topographic effects in force microscopy. J Appl Phys, 67(12):7278-7280, 1990.

29. G. D. Skidmore and E. D. Dahlberg. Improved spatial resolution in magnetic force microscopy. Appl Phys Lett, 71(22):3293-3295, 1997.

30. A. J. Stevenson, M. B. Gray, H.-A. Bachor, and D. E. McClelland. Quantumnoise limited interferometric phase measurements. Applied Optics, 32:3481, 1993. 
31. K. Sueoka, K. Okuda, N. Matsubara, and F. Sai. Study of tip magnetization behavior in magnetic force microscope. Journal of Vacuum Science 83 Technology B, 9(2):1313-1317, 1991.

32. O. Teschke. Micromagnetic structure images taken using platinum coated tips. Applied Physics Letters, 79(17):2773-2775, 2001.

33. B. Vellekoop, E. Abelmann, S. Porthun, and C. Lodder. On the determination of the internal magnetic structure by magnetic force microscopy. J Magn Magn Mater, 190(1-2):148-151, 1998. 



\section{Contents}

Towards higher resolution in Magnetic Force Microscopy

Leon Abelmann, Arnout van den Bos, Cock Lodder ............... 1

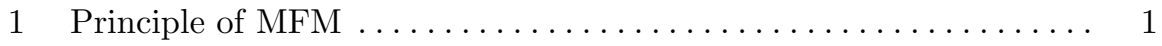

1.1 Mode of operation $\ldots \ldots \ldots \ldots \ldots \ldots \ldots \ldots \ldots \ldots \ldots \ldots \ldots \ldots$

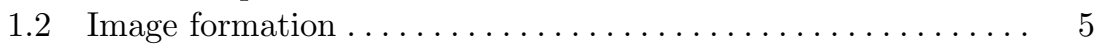

1.3 Instrumentation .............................. 8

2 MFM in Magnetic Data Storage Research $\ldots \ldots \ldots \ldots \ldots \ldots \ldots . \ldots 12$

3 Limits of resolution in MFM ........................ 13

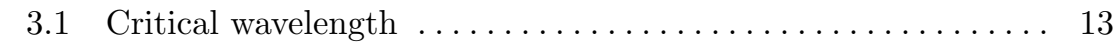

3.2 Thermal noise limited resolution $\ldots \ldots \ldots \ldots \ldots \ldots \ldots \ldots \ldots$

3.2 .1 Thermal Noise ......................... 16

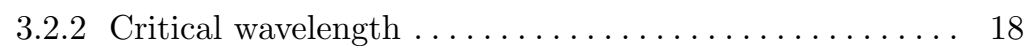

3.2 .3 Beware! ............................ 21

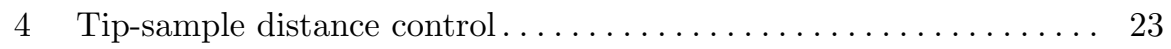

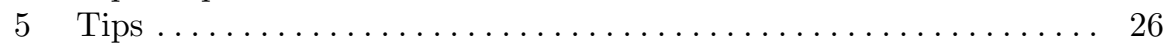

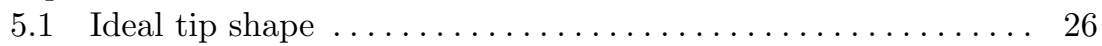

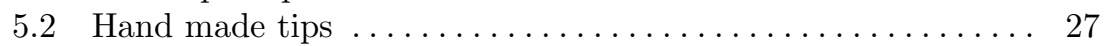

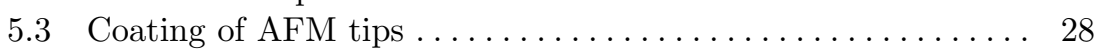

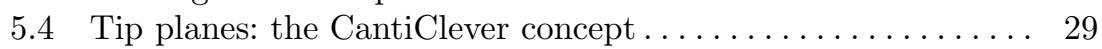

6 Acknowledgement ................................ 33

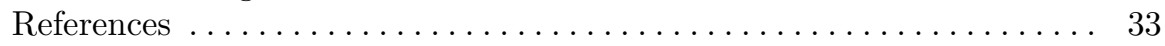

